\title{
Consensus Analysis of Multiagent Networks via Aggregated and Pinning Approaches*
}

\author{
Wenjun Xiong ${ }^{1}$, Daniel W.C. Ho ${ }^{1}$ and Zidong Wang ${ }^{2}$ \\ ${ }^{1}$ Department of Mathematics, City University of Hong Kong, \\ Hong Kong, China. \\ xwenjun2@student.cityu.edu.hk(W. Xiong) \\ madaniel@cityu.edu.hk (D.W.C. Ho) \\ ${ }^{2}$ Department of Information Systems and Computing, Brunel University, \\ Uxbridge, Middlesex, UB8 3PH, United Kingdom. \\ Zidong.Wang@brunel.ac.uk
}

\begin{abstract}
In this paper, the consensus problem of multiagent nonlinear directed networks (MNDNs) is discussed in the case that a MNDN does not have a spanning tree to reach the consensus of all nodes. By using the Lie algebra theory, a linear node-and-node pinning method is proposed to achieve a consensus of a MNDN for all nonlinear functions satisfying a given set of conditions. Based on some optimal algorithms, large-size networks are aggregated to small-size ones. Then, by applying the principle minor theory to the small-size networks, a sufficient condition is given to reduce the number of controlled nodes. Finally, simulation results are given to illustrate the effectiveness of the developed criteria.

Keywords: Directed networks; Absolute consensus; Lie algebra; Graph Laplacian; Pinning consensus; node-and-node pinning method.
\end{abstract}

\section{Introduction}

Over the last decade, the multiagent distributed coordination problem has received much attention in many fields such as biology, physics and engineering. One of the critical research problems is how to control all agents in a network to reach a collective state or an ordered state, such as flocking of birds, schooling of fishes ([1]-[3]). Here, the collective (or ordered) state can be further generalized to consensus, where the term of consensus means to reach an agreement regarding a certain quantity of interest that depends on the states of all agents.

Since the pioneering work stemming from management science and statistics in 1960s (see [4] and the references therein), collective problems have received a great deal of research interests ([5]-[19]). It is shown in [5] that the consensus can be achieved if the union of the interaction graphs is connected frequently enough as the system evolves. In order to extend the results in [5], the work in [6] shows that consensus under dynamically changing interaction topologies can be achieved asymptotically if the union

${ }^{*}$ This work was jointly supported by CityU under a research grant (7002355) and GRF funding (CityU 101109). 
of the directed interaction graphs have a spanning tree frequently. In [7], the authors discuss the averageconsensus problem with directed graph, which requires the graph to be strongly connected and balanced.

It should be pointed out that most of the literature mentioned so far has studied the consensus problem of linear networks. However, in some cases, the state of an agent may be observed to be a nonlinear network. Hence, it is necessary to consider consensus problems of MNDNs. The work in [20] shows that, for some kind of nonlinear networks, the consensus of all nodes can be realized if and only if its graph has a spanning tree. As a result, MNDNs cannot reach a consensus when its graph does not have a spanning tree. Hence, some control schemes have to be designed to force the nonlinear network to achieve a consensus. Clearly, it is meaningful if one can design linear controllers to achieve the consensus of nonlinear networks. Therefore, we shall discuss the consensus of MNDNs by using linear control methods. Moreover, we shall attempt to achieve the absolute consensus for some kind of nonlinear networks. The detailed definition of absolute consensus will be described in Section 2 .

It is costly and impractical to control all nodes in a network. To reduce the number of nodes to be controlled, a pinning scheme is introduced on a fraction of network nodes ([21][22]). Subsequently, large amount of work has been devoted to the study of synchronization or stability of complex networks by using the pinning control (see [23]-[33] and the references therein). In [23], the authors show, both theoretically and numerically, that the pinning scheme is more effective by choosing those nodes with the highest degrees to be controlled. It is demonstrated in [24] that complex networks can achieve a synchronization by pinning a single controller when the coupling strength in the networks is large. As has been revealed in [25], the nodes with low degrees should be controlled first if the coupling strength is not large. Also, in [26], it is shown that the number of controlled nodes cannot be less than $m$ (Here, $m$ is the number of non-negative eigenvalues of a characteristic matrix). In this paper, a principle minor theory in [34] is applied to reduce the number of controlled nodes.

Motivated by the above discussions, the contribution of this paper is presented as follows:

1) A node-and-node linear pinning scheme is first addressed to discuss the consensus of nonlinear networks. In this pinning scheme, some nodes are controlled by some other nodes via directed connections among the nodes.

2) In order to use the method in [34] correctly, we shall aggregate the original network by taking some nodes with similar properties as an aggregated node. The objective of this aggregation is to reduce the number of nodes in the network so that only small-size networks need to be computed.

3) Based on the Lie algebra theory [35; 36], a sufficient condition will be proposed to achieve the absolute consensus of a MNDN via the node-and-node linear pinning scheme.

The remainder of this paper is organized as follows. In Section 2, some definitions and lemmas about directed graph are presented. The Lie algebra theory is addressed and the research problem is described in Section 3. In Section 4, based on some optimal algorithms, large-size networks are aggregated to small-size ones. By using the principle minor theory to small-size networks correctly [34], a node-and-node linear pinning-control sufficient condition is derived to ensure the absolute consensus of the small-size MNDN and reduce the number of controlled nodes in Section 5. In Section 6, simulations are carried out to illustrate the effectiveness of the main results. Finally, conclusions are drawn in Section 7. 


\section{Preliminaries}

Let $\mathcal{G}(\mathcal{V}, \varepsilon, \mathcal{A})$ be a digraph of order $n$ with the set of nodes $\mathcal{V}=\left\{v_{1}, v_{2}, \cdots, v_{n}\right\}, \varepsilon \subseteq \mathcal{V} \times \mathcal{V}$ be the set of edges, and $\mathcal{A}=\left(a_{i j}\right)_{n \times n}$ be a weighted adjacency matrix. An edge of $\mathcal{G}$ is denoted by $e_{i j}=\left(v_{i}, v_{j}\right)$. $e_{i j} \in \varepsilon$ means that there is a directed connection from node $v_{j}$ to node $v_{i}$. The entry $a_{i j}>0$ if $e_{i j} \in \varepsilon$, and $a_{i j}=0$ otherwise. Moreover, it is assumed that $a_{i i}=0$ for all $i \in\{1,2, \cdots, n\}$. The Laplacian of the directed graph is defined as $L=\Delta-\mathcal{A}$, and $\Delta=\left(\Delta_{i j}\right)_{n \times n}$ is a diagonal matrix with $\Delta_{i i}=\sum_{j=1}^{n} a_{i j}$.

In a digraph, a directed path is an ordered sequence of vertices such that any two consecutive vertices are an edge of the digraph. If there is a directed path from every node to the other node, the digraph is said to be strongly connected ([16]). A digraph is undirected ([16]) if $a_{i j}=a_{j i}$ for all $i, j \in\{1,2, \cdots, n\}$. Obviously, the Laplacian of an undirected graph is symmetric. A directed graph is called weakly connected if replacing all of its directed edges with undirected edges produces a connected undirected graph. A digraph $\mathcal{H}$ is a spanning tree ([16]) if it has $m$ vertices and $m-1$ edges and there exists a root vertex with directed paths to all other vertices. A directed tree $\mathcal{H}$ is a spanning tree of a graph $\mathcal{G}$ if $\mathcal{H}$ has the same vertex set as $\mathcal{G}$.

Assume that a network system has $n$ agents, and each agent is regarded as a node in a directed graph $\mathcal{G}$. Let $x_{i}(t) \in R$ denote the state of agent $v_{i}$, then $\mathcal{G}_{x}=(\mathcal{G}, x(t))$ with $x(t)=\left(x_{1}(t), x_{2}(t), \cdots, x_{n}(t)\right)^{T}$ is a directed network. Agents $v_{i}$ and $v_{j}$ in the directed network are said to reach a (an) consensus (agreement) ([7]) if and only if $\left|x_{i}(t)-x_{j}(t)\right| \rightarrow 0$ as $t \rightarrow+\infty$, for all $i, j \in\{1,2, \cdots, n\}, i \neq j$. If the nodes are all in an agreement, the common value $\mathcal{X}(x)$ is called the group decision value $([7])$.

Suppose the multi-agent nonlinear network has the following dynamics

$$
\frac{d x_{i}(t)}{d t}=\sum_{v_{j} \in N_{i}} a_{i j}\left(\breve{f}\left(x_{j}(t)\right)-\breve{f}\left(x_{i}(t)\right)\right), \quad i=1,2, \cdots, n,
$$

where $x_{i}(t)$ is the state of agent $v_{i}, \breve{f}\left(x_{i}(t)\right)$ is a nonlinear function and has the same dimension with $x_{i}(t)$. The dimension of $x_{i}(t)$ could be arbitrary as long as it is the same for all agents. In this paper, for simplification, we only analyze the case when the dimension of $x_{i}(t)$ is one. It is worth noticing that our analysis is valid for any dimension $n$ when the system models are rewritten with Kronecker products.

According to the definition of the Laplacian $L,(1)$ can be rearranged as

$$
\frac{d x(t)}{d t}=-L f(x(t))
$$

where $x=\left(x_{1}(t), x_{2}(t), \cdots, x_{n}(t)\right)^{T}, f(x(t))=\left(\breve{f}\left(x_{1}(t)\right), \breve{f}\left(x_{2}(t)\right), \cdots, \breve{f}\left(x_{n}(t)\right)\right)^{T}$. Clearly, it is difficult and almost impossible to discuss the consensus of system (2) without any limitation on the nonlinear function $f(x(t))$. Hence, we assume that

Assumption 1 Nonlinear function $\breve{f}(\cdot)$ satisfies that $\breve{f}(\cdot) \in S$, where $S$ denotes a set of nonlinear functions, and each $s \in S$ is continuous and strictly increasing. Moreover, for each $\breve{f}(\zeta) \in S$, $\breve{f}(\zeta)=0 \Leftrightarrow \zeta=0, \forall \zeta \in R$. 
Definition 1 The multi-agent network (2) is said to achieve an absolute consensus if it obtains a consensus for every nonlinear function $\breve{f}(\cdot) \in S$.

Throughout the paper, the following Lemmas are needed.

Lemma 1 [37] All eigenvalues of L have non-negative real part. The multiplicity of the zero eigenvalue of $L$ is equal to the minimum number of directed trees which forms a spanning forest in the digraph.

Lemma 2 [20] Under Assumption 1, model (2) achieves a consensus if and only if the directed graph $\mathcal{G}$ has a spanning tree.

Notation: Throughout this paper, $I$ stands for the identity matrix. The superscript " $T$ " represents the transpose. For all $x=\left(x_{1}, x_{2}, \cdots, x_{n}\right)^{T} \in R^{n}$, the notation $\|x\|=\left(\sum_{i=1}^{n} x_{i}^{2}\right)^{\frac{1}{2}}$. For a symmetric matrix $A$, $\lambda_{m}(A)$ and $\lambda_{M}(A)$ denote the minimal and maximal eigenvalues of matrix $A$ respectively. $\|A\|$ denotes the spectral norm defined by $\|A\|=\left(\lambda_{M}\left(A^{T} A\right)\right)^{\frac{1}{2}}$. For real symmetric matrixes $X$ and $Y, X>Y$ (or $X \geq Y$ ) means that matrix $X-Y$ is positive definite (or positive semi-define).

\section{Problem description}

In this paper, we assume that graph $\mathcal{G}$ does not contain a spanning tree, and the agents have different initial values. Then, from Lemma 2, system (2) (or (1)) cannot reach a consensus. As a result, some control methods should be introduced to guarantee the consensus of all agents. In this section, we shall introduce a node-and-node linear pinning strategy in nonlinear system (1). The pinning controlled system of model (1) can be described by

$$
\frac{d x_{i}(t)}{d t}=\sum_{v_{j} \in N_{i}} a_{i j}\left(\breve{f}\left(x_{j}(t)\right)-\breve{f}\left(x_{i}(t)\right)\right)+\sum_{i, j \in \phi} \widetilde{d}_{i j}\left(x_{j}(t)-x_{i}(t)\right), \quad i=1,2, \cdots, n,
$$

where $\phi=\{1,2, \cdots, n\}, \widetilde{D}=\left(\widetilde{d}_{i j}\right)_{n \times n} \in R^{n \times n}$. Here, $\widetilde{d}_{i j} \geq 0$, for all $i, j \in \phi$, and $\widetilde{d}_{i i}=0$, for all $i \in \phi$. For all $i \neq j, i, j \in \phi$, if node $j$ controls node $i$ (that is, there is a directed edge from node $j$ to node $i$ ), then $\widetilde{d}_{i j}>0$; otherwise, $\widetilde{d}_{i j}=0$. Here, the second term on the right-hand side of (3) is called a node-and-node pinning controller since it means that some nodes are directly controlled by some other nodes.

System (3) can be rewritten as

$$
\frac{d x(t)}{d t}=-L f(x(t))-D x(t)
$$

where the node-and-node pinning scheme is given by $u(t) \triangleq-D x(t)$, and $D=\left(d_{i j}\right)_{n \times n} \in R^{n \times n}$. For all

$i \neq j, i, j \in \phi, d_{i j}=-\widetilde{d}_{i j}$, and $d_{i i}=\sum_{j=1, j \neq i}^{n} \widetilde{d}_{i j}$. Here, matrix $D$ is the Laplacian matrix of matrix $\widetilde{D}$. As a result, the row sum of matrix $D$ is also zero. 
Remark 1 In the existing literature [24; 25; 29] about pinning control, the authors obtain the error system of a complex network, and then pin the error system with a controller whose matrix is required to be diagonal. That is, external control actions are forced on some nodes. However, different from previous work, the corresponding matrix $D$ of controller $u(t)$ is not required to be diagonal. That is, based on the graph theory and the structure of the discussed network, some nodes are directly controlled by some other nodes via the directed connections among the nodes.

Before proceeding further, some definitions and preliminaries about Lie algebra theory [35] are first introduced.

A vector space $\mathfrak{L}$ of $n \times n$ matrices is called as a matrix Lie algebra if the commutator product $[A, B]=A B-B A \in \mathfrak{L}$, for any $A, B \in \mathfrak{L}$.

Definition 2 [35] A set of $n \times n$ matrices $\left\{B_{1}, B_{2}, \cdots, B_{m}\right\}$ is said to generate a Lie algebra $\mathfrak{L}$ if $\mathfrak{L}=$ $\operatorname{span}\left\{B_{1}, B_{2}, \cdots, B_{m}\right\}$, where $m$ is a positive integer.

For a Lie algebra $\mathfrak{L}$, define the inductive sequence:

$$
\mathfrak{L}^{(0)}=\mathfrak{L}, \quad \mathfrak{L}^{(i+1)}=\left\{[A, B]: A, B \in \mathfrak{L}^{(i)}\right\}, \quad i=0,1,2, \cdots
$$

It is easy to verity that $\mathfrak{L}^{(i)}$ is well-defined.

Definition 3 [35] The matrix Lie algebra $\mathfrak{L}$ is called solvable if there exists an integer $p>0$ such that

$$
\mathfrak{L}^{(p)}=\{0\}
$$

If $\mathfrak{L}$ is a matrix Lie algebra of a set of pairwise commuting matrices, i.e., matrices $A, B$ satisfy that $A B=B A$, for all $A, B \in \mathfrak{L}$, we have $\mathfrak{L}^{(1)}=\{0\}$. That is, the solvable matrix Lie algebra always exists. Question $Q_{1}$ has arisen: can condition (5) be satisfied for some $p \leq n$ if $\mathfrak{L}$ is a solvable Lie algebra of $n \times n$ matrices? In order to answer this problem, we introduce the following Lemma.

Lemma 3 [36] A matrix Lie algebra $\mathfrak{L}$ is solvable if and only if there exists a nonsingular matrix $T$ such that $T^{-1} A T$ is upper triangular for all $A \in \mathfrak{L}$.

From Lemma 3, it follows that the solvability condition (5) can be verified within finite steps $p$, where $p \leq n$ and $n$ is the order of the matrices. The corresponding detailed description can be seen in the Appendix. Hence, an answer is given for the aforementioned question $Q_{1}$.

Lemma 4 [36] A solvable matrix Lie algebra is unitarily equivalent to an upper triangular matrix Lie algebra, i.e., matrix $T$ in Lemma 3 can be chosen to be a unitary matrix. Here, if matrix $T$ is a real matrix, $T$ is called as an orthogonal matrix. 
For all matrices $A \in R^{n \times n}$, define

$$
A_{1}=\frac{1}{2}\left(A+A^{T}\right), \quad A_{2}=\frac{1}{2}\left(A-A^{T}\right)
$$

Then, $A=A_{1}+A_{2}$, and $A_{1}, A_{2}$ are the symmetric and skew-symmetric parts of $\mathrm{A}$. The following result can be obtained from Lemma 3 and Lemma 4.

Lemma 5 [36] Suppose $A$ is a real matrix in $R^{n \times n}$. If $\left\{A_{1}, A_{2}\right\}$ generates a solvable Lie algebra, we have

$$
\{\operatorname{Re} \lambda(A)\}=\left\{\lambda\left(A_{1}\right)\right\}
$$

where, $\lambda(A)$ is an eigenvalue of matrix $A$ and $\operatorname{Re} \lambda(A)$ is the real part of $\lambda(A)$.

Remark 2 If a matrix $A \in R^{n \times n}$ is normal, i.e., $A^{T} A=A A^{T}$, it is easy to obtain that $A_{1} A_{2}=A_{2} A_{1}$. As a result, $\left\{A_{1}, A_{2}\right\}$ generates a solvable Lie algebra. In addition, if a matrix $A \in R^{n \times n}$ is symmetric, i.e., $A=A^{T}$, then $\left\{A_{1}, A_{2}\right\}$ also generates a solvable Lie algebra. Hence, the condition that $\left\{A_{1}, A_{2}\right\}$ generates a solvable Lie algebra is not difficult to be achieved in practice.

Lemma 6 Let $G_{1}$ be a maximal-order positive-definite principle minor of a $k$-order symmetric matrix $G$, and $\operatorname{rank}\left(G_{1}\right)=k-l(0 \leq l \leq k)$. If one wants to use a diagonal matrix $C=\operatorname{diag}\left(c_{1}, c_{2}, \cdots, c_{k}\right)\left(c_{i} \geq 0\right.$ is appropriately large) to guarantee

$$
G+C>0
$$

then the rank of matrix $C$ is at least $l$ (i.e., $p \geq l$ ), $p \geq 0$ is an integer and denotes the total number of non-zero elements in matrix $C$. Matrix $C$ is called the pinning controller of $G$. Actually, the $l$ nodes which are not in the rows (or columns) of $G_{1}$ can be pinned to obtain (7).

Proof: one can obtain the result by using the same method in Theorem 2 of [34]. The proof is omitted here. The Algorithm is shown in Appendix B.

Remark 3 In Proposition 7 of [26], the authors concluded that, if $G$ in (7) has $w$ ( $w$ is an integer and $0 \leq w \leq n$ ) non-positive eigenvalues, the rank of matrix $C$ is not less than $w$. However, it is not sufficient to obtain (7) if $p=w$. For example, in (7), if $G=\left(\begin{array}{ccc}1 & -1 & -2 \\ -1 & 0 & -1 \\ -2 & -1 & 1\end{array}\right)$, the number of non-positive eigenvalues of $G$ is 1 . However, if $p=1$, one cannot achieve (7) even though $C$ is chosen to be very large. The number of maximal order positive-definite principle minor of matrix $G$ is 1 . According to some simple calculations, (7) can be achieved if $p=2$ (such as $c_{1}=0, c_{2}>0, c_{3}>0$ ). This example shows that the result in Lemma 6 is more effective than that in [26].

Next, an aggregated method is presented to reduce the number of nodes in the network so that only small-size networks need to be computed. 


\section{Aggregation approach}

It is worth noting that the result in Lemma 6 is effective to compute for a small-size complex network but not for a large-size one. Therefore, by using some optimal algorithms, the original network of large size can be aggregated by taking those nodes with similar properties and, therefore, as an aggregated node. The objective of this aggregation is to effectively reduce the number of nodes in the network so that only small-size networks are considered.

Since graph $\mathcal{G}$ does not contain a spanning tree, there exist at least two root nodes which have no information transmission to each other. In graph $\mathcal{G}$, those root nodes are called leaders, and other nodes are called followers. Then, we state the following general Property.

Property 1 For system (2) (or(1)), the leaders have to decide whether system (2) (or (1)) reaches a consensus and also the group decision value (if a consensus can be obtained). Those followers may affect the convergent rate of system (2) (or (1)), but they do not affect the final consensus (or un-consensus) status.

Remark 4 We shall explain this property via the graph theory. For any leader with one of its followers in system (2) (or(1)), their connections can be seen in Fig. 1. One can see that the followers always receive the leaders' information but the leaders do not receive any information from the followers. Moreover, according to the received information, the followers adjust their state values to obtain the consensus with the state values of leaders. In principle, the leaders have to decide two issues: i) whether system (2) (or (1)) reaches a consensus, ii) the group decision value (if a consensus can be obtained). In contrast, the followers do not affect the above two issues.

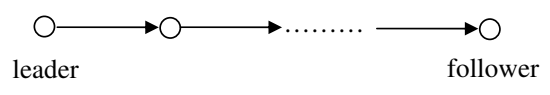

Figure 1: The connection between a leader and a follower.

In addition, when a follower receive its leaders' information and then adjust its own state value, the required time depends on the numbers of followers and the distances between the leaders and the followers. Hence, the followers will affect the convergent rate of system (2) (or (1)).

Now, we shall aggregate the graph of system (2) (or (1)) by using a modified method in [38]. For the original graph $\mathcal{G}(\mathcal{V}, \varepsilon)$, construct the aggregated graph $\widetilde{\mathcal{G}}(\widetilde{\mathcal{V}}, \widetilde{\varepsilon})$ as follows:

a) The node set is given by $\widetilde{V}=\{1,2, \cdots, \widetilde{n}\}$, and each node $i$ represents a partition set $U_{i}$ of $\mathcal{V}$. That is, $\bigcup_{i} U_{i}=\mathcal{V}$ and $U_{i} \cap U_{j}=\emptyset, \forall i \neq j$. We call the set $U_{i}$ a group of nodes.

b) The edge set $\widetilde{\varepsilon}=\widetilde{V} \times \widetilde{V}$ satisfies that if $\left(i_{1}, i_{2}\right) \in \varepsilon$, then $\left(h\left(i_{1}\right), h\left(i_{2}\right)\right) \in \widetilde{\varepsilon}$, where $h: \varepsilon \rightarrow \widetilde{\varepsilon}$ is the function indicating the group $j$ that node $i$ belong to such that $h(i)=j$, or $i \in U_{j}$.

Compared with the method in [38], the difference is that our aggregated process is implemented based on the following two points: 
I) In any partition set $U_{i}, i \in \widetilde{V}$, we restrict that there has only a root node.

II) Note that there may exist two or more leaders for a follower $i$. We only choose one leader $j$ and then let the follower $i$ belong to $U_{j}$ (i.e., $i \in U_{j}$ ).

Remark 5 The properties of our aggregation method are summarized as follows:

The purpose of this aggregation is to regroup those followers with one of their leaders (root nodes). There may be many different combinations of regrouping partition sets when a follower chooses a different leader. However, the values of the states of different partition sets are still the same when they have a same root node. The reasons are as follows:

i) Those followers do not affect the consensus (or un-consensus) status of the partition sets (see Property 1);

ii) The values of these partition sets are decided by the root nodes (see Property 1);

iii) The roots nodes are fixed when the network model (1) is given.

iv) Each new node in the aggregated network contains the same leader with other different choices of followers.

Therefore, one can say that the aggregated network is independent of the choice of the re-grouping of nodes. However, the convergent rate of the aggregated network may be different for different choices of re-grouping.

Remark 6 According to Property 1, one knows that the model of the aggregated graph has the same consensus (or un-consensus) status with that of the original graph. The only difference may be the convergent rate of consensus. As a result, it is reasonable to discuss the consensus property of the aggregated system instead of the original system. Actually, compared with [38], the advantage of our modified method is that there is no error between the final decision value (i.e., the final vector value of a system) of the original graph $\mathcal{G}(\mathcal{V}, \varepsilon)$ and that of the aggregated graph $\widetilde{\mathcal{G}}(\widetilde{\mathcal{V}}, \widetilde{\varepsilon})$. The only difference is the convergent rate, i.e., the time needed to obtain the final decision value.

We define the weighted matrix of the aggregated graph $\widetilde{\mathcal{G}}(\widetilde{\mathcal{V}}, \widetilde{\varepsilon})$ is $\widetilde{A}$, and $\widehat{L}$ is the corresponding laplacian of matrix $\widetilde{A}$. Now, we present a simple example to address how to aggregate a system.

Example 1 Consider the graph consisting seven nodes shown in the left graph (a) of Fig. 2. From the graph, it is easy to see that nodes 1, 2, 3, 7 are leaders, i.e., root nodes. Nodes 4 and 5 are the followers of node 3. Node 6 is the follower of node 1 and node 3. We partition the nodes as $U_{1}=\{1,6\}, U_{2}=$ $\{2\}, U_{3}=\{3,4,5\}, U_{4}=\{7\}$ as indicated by the dashed lines. Then, the aggregated graph can be obtained in the right graph (b) of Fig. 2.

Let all of the connection weights be 1 . The laplacian matrices $L$ and $\widehat{L}$ of graphs $(a)$ and $(b)$ are as 
follows

$$
L=\left(\begin{array}{ccccccc}
1 & 0 & -1 & 0 & 0 & 0 & 0 \\
-1 & 1 & 0 & 0 & 0 & 0 & 0 \\
0 & -1 & 1 & 0 & 0 & 0 & 0 \\
0 & 0 & -1 & 1 & 0 & 0 & 0 \\
0 & 0 & 0 & -1 & 1 & 0 & 0 \\
-1 & 0 & -1 & 0 & 0 & 2 & 0 \\
0 & 0 & 0 & 0 & 0 & 0 & 0
\end{array}\right), \widehat{L}=\left(\begin{array}{cccc}
1 & 0 & -1 & 0 \\
-1 & 1 & 0 & 0 \\
0 & -1 & 1 & 0 \\
0 & 0 & 0 & 0
\end{array}\right)
$$

Let $f(x(t))=\left(\breve{f}\left(x_{1}(t)\right), \breve{f}\left(x_{2}(t)\right), \cdots, \breve{f}\left(x_{7}(t)\right)\right)^{T}$ with $\breve{f}\left(x_{i}(t)\right)=x_{i}^{3}(t), \quad i=1,2, \cdots, 7$. Let the initial value of nodes in graph $(a)$ be $(-1,2,3,1,5,-2,4)^{T}$. By writing some simple computer programs based on the MATLAB Toolbox, one can obtain that the final decision value of nodes in graph $(a)$ is $(1.3333,1.3333,1.3333,1.3333,1.3333,1.3333,4)^{T}$. In graph $(b)$, since nodes 1, 2, 3, 7 are leaders, then the initial value of set $\left\{U_{1}, U_{2}, U_{3}, U_{4}\right\}$ are assigned as the initial value of nodes 1, 2, 3, 7, i.e., $\left(U_{1}, U_{2}, U_{3}, U_{4}\right)^{T}=(-1,2,3,4)^{T}$. Similarly, by using the same MATLAB program, one can obtain that the final decision value of set $\left\{U_{1}, U_{2}, U_{3}, U_{4}\right\}$ in graph $(b)$ is $(1.3333,1.3333,1.3333,4)^{T}$. Since nodes 1 , 2, 3, 7 are leaders, correspondingly, one can obtain that the final decision value of seven nodes in the original network is also $(1.3333,1.3333,1.3333,1.3333,1.3333,1.3333,4)^{T}$. Hence, we say that there is no error between the final decision value of the original graph $\mathcal{G}(\mathcal{V}, \varepsilon)$ and that of the aggregated graph $\widetilde{\mathcal{G}}(\widetilde{\mathcal{V}}, \widetilde{\varepsilon})$.

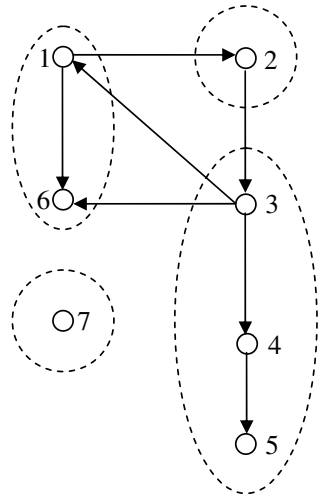

(a)

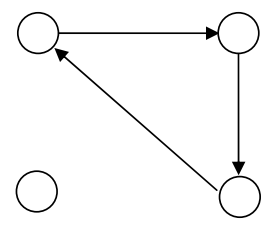

(b)

Figure 2: The left graph $(a)$ is the original graph, and the right graph $(b)$ is the aggregated graph.

For the small-size networks like Example 1, it is easy to find out the root nodes and then obtain the aggregated graph. However, it is difficult to find out all the root nodes to obtain the aggregated graph for a large-size network. Hence, an algorithm is proposed to illustrate how to obtain the aggregated graph effectively for a large-size network.

Algorithm 1 For the Laplacian matrix $L=\left(l_{i j}\right)_{n \times n}$ of the original graph $\mathcal{G}(\mathcal{V}, \varepsilon)$, we implement the following three steps:

1) Check the columns of matrix L. If there has only one nonzero element $l_{i j}$ in a column, then we delete the ith row and the $j$ th column of matrix $L$. 
2) Repeat the above process until one cannot find a column which has only one nonzero element.

3) The retained matrix is matrix $\widehat{L}$. Correspondingly, one can get the aggregated graph $\widetilde{\mathcal{G}}(\widetilde{\mathcal{V}}, \widetilde{\varepsilon})$.

Remark 7 Note that the above aggregation method is only effective for a large-size network with small numbers of root nodes but not for a general large-size one. We attempted to use the same aggregated method in [38] to aggregate a general large-size network to a small-size one. Unfortunately, we found that the error between the final decision value of the original network and that of the aggregated one is big. We have to say that our proposed method is crude at this stage, but it thus provides some insights to the aggregated problem. Hence, it is still an open problem to find an appropriate method with small (or without) errors to aggregate a general large-size network.

In this section, we have proposed an aggregation method to reduce the number of nodes in large-size multiagent networks. Next, we shall discuss the absolute consensus of the aggregated model of system (2) based on Lemma 6 and the node-and-node pinning method.

\section{Absolute consensus of MNDNs based on Lie algebra}

The aggregated model of system (2) can be rearranged as

$$
\frac{d \widehat{x}(t)}{d t}=-\widehat{L} \widehat{f}(\widehat{x}(t))
$$

where $\widehat{x}(t)=\left(\widehat{x}_{1}(t), \widehat{x}_{2}(t), \cdots, \widehat{x}_{\widetilde{n}}(t)\right)^{T}$, and $\widehat{x}_{i}(t) \in R(i \in\{1,2, \cdots, \widetilde{n}\})$ is the state of set $U_{i}$ in graph $\widetilde{\mathcal{G}}(\widetilde{\mathcal{V}}, \widetilde{\varepsilon}) \cdot \widehat{f}(\widehat{x}(t))=\left(\breve{f}\left(\widehat{x}_{1}(t)\right), \breve{f}\left(\widehat{x}_{2}(t)\right), \cdots, \breve{f}\left(\widehat{x}_{\widetilde{n}}(t)\right)\right)^{T}$ The node-and-node linear pinning controlled system of model (8) can be described by

$$
\frac{d \widehat{x}(t)}{d t}=-\widehat{L} \widehat{f}(\widehat{x}(t))-\widehat{D} \widehat{x}(t)
$$

where the node-and-node pinning scheme is given by $\widehat{u}(t) \triangleq-\widehat{D} \widehat{x}(t)$, and $\widehat{D}=\left(\widehat{d}_{i j}\right)_{\tilde{n} \times \tilde{n}} \in R^{\tilde{n} \times \widetilde{n}}$. Here, matrix $\widehat{D}$ has the similar definition with matrix $D$ of (4). That is, the row sum of matrix $\widehat{D}$ is also zero.

In the following, we shall address the absolute consensus of system (9). Without loss of generality, we assume that the consensus means that all other $\widetilde{n}-1$ nodes move to the position of node $\widetilde{n}$ respectively in this paper.

To model the above phenomena, let $y(t)=Q \widehat{x}(t)$ with matrix $Q=\left(\begin{array}{ccccc}1 & 0 & \cdots & 0 & -1 \\ 0 & 1 & \cdots & 0 & -1 \\ \cdots & \cdots & \cdots & \cdots & \cdots \\ 0 & 0 & \cdots & 1 & -1 \\ 0 & 0 & \cdots & 0 & 1\end{array}\right)_{\widetilde{n} \times \widetilde{n}}$, then $y(t)=\left(\widehat{x}_{1}(t)-\widehat{x}_{\widetilde{n}}(t), \widehat{x}_{2}(t)-\widehat{x}_{\widetilde{n}}(t), \cdots, \widehat{x}_{\tilde{n}-1}(t)-\widehat{x}_{\widetilde{n}}(t), \widehat{x}_{\tilde{n}}(t)\right)^{T}$. System $(9)$ can be rewritten as

$$
\frac{d y(t)}{d t}=-Q \widehat{L} Q^{-1} Q \widehat{f}(\widehat{x}(t))-Q \widehat{D} Q^{-1} y(t)
$$


Let $z(t)=\left(z_{1}(t), z_{2}(t), \cdots, z_{n-1}(t)\right)^{T} \triangleq\left(\widehat{x}_{1}(t)-\widehat{x}_{\widetilde{n}}(t), \widehat{x}_{2}(t)-\widehat{x}_{\tilde{n}}(t), \cdots, \widehat{x}_{\tilde{n}-1}(t)-\widehat{x}_{\tilde{n}}(t)\right)^{T}$, then one has $y(t)=\left(z^{T}(t), \widehat{x}_{\tilde{n}}(t)\right)^{T}$. Hence, a consensus of all agents is reached if one obtains $z(t) \rightarrow 0$ as $t \rightarrow+\infty$. That is, $\widehat{x}_{i}(t) \rightarrow \widehat{x}_{\widetilde{n}}(t)$ if $z(t) \rightarrow 0$ as $t \rightarrow+\infty, i=1,2, \cdots, \widetilde{n}-1$.

Remark 8 Without loss of generality, we assume that the group decision value is $\widehat{x}_{\tilde{n}}$ in system (9). Actually, different group decision value can be obtained if different transformation matrix $Q$ is chosen. For example, if $Q=\left(\begin{array}{ccccc}1 & 0 & \cdots & 0 & 0 \\ -1 & 1 & \cdots & 0 & 0 \\ \cdots & \cdots & \cdots & \cdots & \cdots \\ -1 & 0 & \cdots & 1 & 0 \\ -1 & 0 & \cdots & 0 & 1\end{array}\right)_{\widetilde{n} \times \widetilde{n}}$, then a consensus embodies that the other $\widetilde{n}-1$ nodes

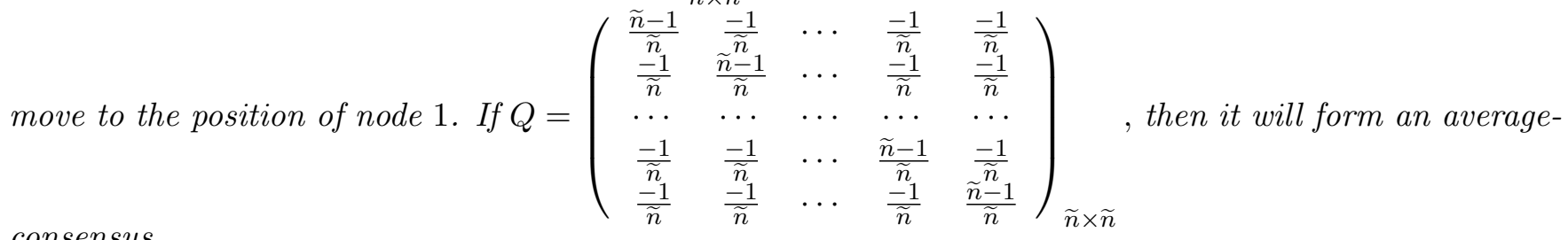
consensus.

According to the property of Laplacian matrix, system (10) can be written as

$$
\left(\begin{array}{c}
d z(t) / d t \\
d \widehat{x}_{\widetilde{n}}(t) / d t
\end{array}\right)=-\left(\begin{array}{cc}
\widetilde{L} & 0 \\
L_{1} & 0
\end{array}\right)\left(\begin{array}{c}
g(z(t)) \\
\breve{f}\left(\widehat{x}_{\widetilde{n}}(t)\right)
\end{array}\right)-\left(\begin{array}{cc}
\bar{D} & 0 \\
D_{1} & 0
\end{array}\right)\left(\begin{array}{c}
z(t) \\
\widehat{x}_{\widetilde{n}}(t)
\end{array}\right)
$$

where $Q \widehat{f}(\widehat{x}(t)) \triangleq\left(g^{T}(z(t)), \breve{f}\left(\widehat{x}_{\widetilde{n}}(t)\right)\right)^{T}$, with $g(z(t))=\left(\breve{g}\left(z_{1}(t)\right), \breve{g}\left(z_{2}(t)\right), \cdots, \breve{g}\left(z_{\widetilde{n}-1}(t)\right)\right)^{T}=\left(\breve{f}\left(\widehat{x}_{1}(t)\right)-\right.$ $\left.\breve{f}\left(\widehat{x}_{\widetilde{n}}(t)\right), \breve{f}\left(\widehat{x}_{2}(t)\right)-\breve{f}\left(\widehat{x}_{\widetilde{n}}(t)\right), \cdots, \breve{f}\left(x_{\widetilde{n}-1}(t)\right)-\breve{f}\left(\widehat{x}_{\widetilde{n}}(t)\right)\right)^{T}, \widehat{L}=\left(\widehat{l}_{i j}\right)_{\tilde{n} \times \tilde{n}}, \widetilde{L}=\left(\widehat{l}_{i j}-\widehat{l}_{\widetilde{n} j}\right)_{(\widetilde{n}-1) \times(\widetilde{n}-1)}$, and $L_{1}=$ $\left(\widehat{l}_{\tilde{n} 1}, \widehat{l}_{\tilde{n} 2}, \cdots, \widehat{l}_{\widetilde{n}(\tilde{n}-1)}\right)^{T}, \bar{D}=\left(\bar{d}_{i j}\right)_{(\widetilde{n}-1) \times(\widetilde{n}-1)}=\left(\widehat{d}_{i j}-\widehat{d}_{\tilde{n} j}\right)_{(\widetilde{n}-1) \times(\widetilde{n}-1)}$, and $D_{1}=\left(\widehat{d}_{\widetilde{n} 1}, \widehat{d}_{\tilde{n} 2}, \cdots, \widehat{d}_{\widetilde{n}(\widetilde{n}-1)}\right)^{T}$. One can obtain from (11)

$$
\frac{d z(t)}{d t}=-\widetilde{L} g(z(t))-\bar{D} z(t)
$$

As mentioned above, in this paper, the consensus means that all other $\widetilde{n}-1$ nodes move to the position of node $\widetilde{n}$ respectively. That is, the state of node $\widetilde{n}$ is the final consensus state. Naturally, it is very effective if the connections in the node-and-node pinning strategy are constructed to be directed edges from node $n$ to other nodes. Hence, for matrix $\widehat{D}=\left(\widehat{d}_{i j}\right)_{\tilde{n} \times \tilde{n}}$, we define that

$$
\begin{cases}\widehat{d}_{i j}=0, & i \neq j, i, j=1,2, \cdots, \widetilde{n}-1 \\ \widehat{d}_{i \widetilde{n}} \leq 0, \widehat{d}_{i i}=-\widehat{d}_{i \widetilde{n}}, & i=1,2, \cdots, \widetilde{n}-1 \\ \widehat{d}_{\widetilde{n} j}=0, & j=1,2, \cdots, \widetilde{n}\end{cases}
$$

As a result, matrix $\bar{D}$ is diagonal and $\bar{d}_{i i}=\widehat{d}_{i i} \geq 0$, for $i=1,2, \cdots, \widetilde{n}-1$.

Model (12) is said to be absolutely stable if it is globally asymptotically stable for every function $\breve{f}(\cdot) \in S$. Clearly, system (4) can obtain an absolute consensus if model (12) is absolutely stable. Hence, we shall discuss the absolute stability of model (12) in the following. 
Remark 9 Note that, the graph $\mathcal{G}$ does not contain a spanning tree in this paper. That is, at least two eigenvalues of $\widehat{L}$ are zero and other eigenvalues have positive real-parts according to Lemma 1. From (11) and (12), one knows that $\widehat{L}$ has the same eigenvalues with $\widetilde{L}$ except for a zero eigenvalue. Hence, all eigenvalues of $\widetilde{L}$ have non-negative real-parts.

Theorem 1 Suppose $\left\{\widetilde{L}_{1}, \widetilde{L}_{2}\right\}$ generates a solvable Lie algebra and $\breve{f}(\zeta)$ is differentiable with the derivative $\breve{f}^{\prime}(\zeta) \leq 1, \forall \zeta \in R$. Then the absolute stability of system (12) can be achieved if

$$
\operatorname{Re}_{1 \leq i \leq \widetilde{n}-1} \lambda_{i}(\widetilde{L}+\bar{D})>0
$$

Proof: Since $g(z(t))=\left(\breve{g}\left(z_{1}(t)\right), \breve{g}\left(z_{2}(t)\right), \cdots, \breve{g}\left(z_{\widetilde{n}-1}(t)\right)\right)^{T}=\left(\breve{f}\left(\widehat{x}_{1}(t)\right)-\breve{f}\left(\widehat{x}_{\widetilde{n}}(t)\right), \breve{f}\left(\widehat{x}_{2}(t)\right)-\breve{f}\left(\widehat{x}_{\widetilde{n}}(t)\right), \cdots\right.$, $\left.\breve{f}\left(x_{\widetilde{n}-1}(t)\right)-\breve{f}\left(\widehat{x}_{\widetilde{n}}(t)\right)\right)^{T}$, and $\breve{f}(\cdot) \in S$. One has that $\breve{g}(\cdot) \in S$. That is, $\breve{g}(\cdot)$ satisfies the conditions in Assumption 1. Hence, one can construct the following Lyapunov function

$$
V(z(t))=\sum_{i=1}^{\widetilde{n}-1} \int_{0}^{z_{i}(t)} \breve{g}(r) d r .
$$

Clearly, $V(z(t))$ is positive definite and radially unbounded. The time derivative of $V(z(t))$ along the solution of system (12) is

$$
\begin{aligned}
\frac{d V(z(t))}{d t} & =-g^{T}(z(t)) \widetilde{L} g(z(t))-g^{T}(z(t)) \bar{D} z(t) \\
& =-g^{T}(z(t)) \widetilde{L}_{1} g(z(t))-g^{T}(z(t)) \bar{D} z(t) \\
& =-g^{T}(z(t))\left(\widetilde{L}_{1}+\bar{D}\right) g(z(t))-g^{T}(z(t)) \bar{D}(z(t)-g(z(t))) .
\end{aligned}
$$

According to Lemma 5 and (14), one obtains that $\widetilde{L}_{1}+\bar{D}>0$. Since $\breve{g}(\cdot) \in S$, one has

$$
g^{T}(z(t))\left(\widetilde{L}_{1}+\bar{D}\right) g(z(t))>0, \forall z(t) \neq 0
$$

Let function $h(\zeta)=\zeta-\breve{f}(\zeta), \forall \zeta \in R$. Since $\breve{f}^{\prime}(\zeta) \leq 1, \forall \zeta \in R$, then one has $h^{\prime}(\zeta)=1-\breve{f}^{\prime}(\zeta) \geq 0$, which means that $h(\cdot)$ is an increasing function. Furthermore, one has $h(0)=0$ according to Assumption 1. Hence, $h\left(z_{i}(t)\right) \triangleq h\left(\widehat{x}_{i}(t)\right)-h\left(\widehat{x}_{\widetilde{n}}(t)\right)=\widehat{x}_{i}(t)-\breve{f}\left(\widehat{x}_{i}(t)\right)-\left(\widehat{x}_{\widetilde{n}}(t)-\breve{f}\left(\widehat{x}_{\widetilde{n}}(t)\right)\right)=z_{i}(t)-\breve{g}\left(z_{i}(t)\right) \geq 0$ when $z_{i}(t) \geq 0, i=1,2, \cdots, \widetilde{n}-1$. As a result, the function $H(z(t)) \triangleq z(t)-g(z(t))$ is also an increasing function and $H(z(t))=0 \Leftrightarrow z(t)=0$. Since matrix $\bar{D} \geq 0$ is diagonal, one has

$$
g^{T}(z(t)) \bar{D}(z(t)-g(z(t))) \geq 0, \forall z(t) \neq 0 .
$$

Hence, $\frac{d V(z(t))}{d t}<0$, for all $z(t) \neq 0$. This shows the absolute stability of model (12). As a result, the absolute consensus of system (4) is achieved. The proof is completed.

Theorem 2 Suppose $\breve{f}(\zeta)$ is differentiable with the derivative $\breve{f}^{\prime}(\zeta) \leq 1, \forall \zeta \in R$. Let the order of a maximal-order positive-definite principle minor of matrix $\widetilde{L}_{1}$ be $n-1-\gamma(0 \leq \gamma \leq n-1)$, then the absolute stability of system (12) can be achieved if the diagonal matrix $\bar{D} \geq 0$ is chosen with $\operatorname{rank}(\bar{D}) \geq \gamma$. 
Proof: Construct the same Lyapunov function with (15), one has

$$
\frac{d V(z(t))}{d t}=-g^{T}(z(t))\left(\widetilde{L}_{1}+\bar{D}\right) g(z(t))-g^{T}(z(t)) \bar{D}(z(t)-g(z(t)))
$$

Note that matrix $\widetilde{L}_{1}$ is symmetric and the order of a maximal-order positive-definite principle minor of $\widetilde{L}_{1}$ is $\widetilde{n}-1-\gamma$. One can let $Q$ be a maximal-order positive-definite principle minor of $\widetilde{L}_{1}$ and the the row (or column) numbers of $Q$ in $G$ be $l_{1}, l_{2}, \cdots, l_{\widetilde{n}-1-\gamma}$. Let $\bar{D}=\left(\bar{d}_{i j}\right)_{(\widetilde{n}-1) \times(\widetilde{n}-1)}$ be a diagonal matrix with

$$
\bar{d}_{i i}\left\{\begin{array}{cc}
=0, & i \in\left\{l_{1}, l_{2}, \cdots, l_{\widetilde{n}-1-\gamma}\right\} \\
>0, & \text { otherwise }
\end{array}\right.
$$

If $\bar{d}_{i i}>0\left(\forall i \in\{1,2, \cdots, \widetilde{n}-1\}-\left\{l_{1}, l_{2}, \cdots, l_{\widetilde{n}-1-\gamma}\right\}\right)$ is appropriately large, one can obtain $\widetilde{L}_{1}+\bar{D}>0$ according to Lemma 6 . Since $\breve{g}(\cdot) \in S$, one has $g^{T}(z(t))\left(\widetilde{L}_{1}+\bar{D}\right) g(z(t))>0, \forall z(t) \neq 0$.

Then, with the similar analysis in Theorem 1 , one obtain $\frac{d V(z(t))}{d t}<0$, for all $z(t) \neq 0$. This shows the absolute stability of model (12). As a result, the absolute consensus of system (4) is achieved. The proof is completed.

Remark 10 The consensus problem in a multi-agent system with general nonlinear coupling has been thoroughly investigated in [33]. It has been clearly demonstrated in [33] that, under suitable conditions on communication, all agents would approach a prescribed value if a small fraction of them are controlled by a simple feedback control. This paper studies the similar problem from a different angle and therefore our results complement those of [33]. The distinguishing features of this paper can be stated as follows:

1) A linear node-and-node pinning method is used in our paper. By using the Lie algebra theory and a completely new pinning method, an absolute consensus of a MNDN is achieved for all nonlinear functions satisfying a given set of conditions in Assumption 1.

2) Based on Lemma 6 and Algorithm 1, our focus of this paper is mainly on discussing the least number of nodes required to be controlled to reach the absolute consensus of a MNDN according to the principle minor of a matrix.

\section{An illustrative example}

In this section, a numerical example is presented to demonstrate the effectiveness of our results on Theorem 2.

Example 2 In this example, a network with 74 nodes is considered based on system (2). Moreover, $f(\widehat{x}(t))=\left(x_{1}(t)+\tanh \left(x_{1}(t)\right), x_{2}(t)+\tanh \left(x_{2}(t)\right), \cdots, x_{74}(t)+\tanh \left(x_{74}(t)\right)\right)^{T}$.

In the given network, we assume that the original graph $\mathcal{G}(\mathcal{V}, \varepsilon)$ can be aggregated to be graph $\widetilde{\mathcal{G}}(\widetilde{\mathcal{V}}, \widetilde{\varepsilon})$ by using Algorithm 1. Graph $\widetilde{\mathcal{G}}(\widetilde{\mathcal{V}}, \widetilde{\varepsilon})$ includes 8 nodes and the corresponding structure can be seen in Fig. 3. Since the original graph $\mathcal{G}(\mathcal{V}, \varepsilon)$ and the aggregated graph $\widetilde{\mathcal{G}}(\widetilde{\mathcal{V}}, \widetilde{\varepsilon})$ has the same consensus (or un-consensus) 
status, we only need to discuss the node-to-node linear pinning control of the aggregated model.

Based on Fig. 3, for models (8) and (12), one has

$$
\widehat{L}=\left(\begin{array}{cccccccc}
3 & -2 & -1 & 0 & 0 & 0 & 0 & 0 \\
-2 & 2 & 0 & 0 & 0 & 0 & 0 & 0 \\
-1 & 0 & 6 & -3 & -2 & 0 & 0 & 0 \\
0 & 0 & -3 & 3 & 0 & 0 & 0 & 0 \\
0 & 0 & -2 & 0 & 2 & 0 & 0 & 0 \\
0 & 0 & 0 & 0 & 0 & 1 & -1 & 0 \\
0 & 0 & 0 & 0 & 0 & -1 & 1 & 0 \\
0 & 0 & 0 & 0 & 0 & 0 & 0 & 0
\end{array}\right), \widetilde{L}=\left(\begin{array}{ccccccc}
3 & -2 & -1 & 0 & 0 & 0 & 0 \\
-2 & 2 & 0 & 0 & 0 & 0 & 0 \\
-1 & 0 & 6 & -3 & -2 & 0 & 0 \\
0 & 0 & -3 & 3 & 0 & 0 & 0 \\
0 & 0 & -2 & 0 & 2 & 0 & 0 \\
0 & 0 & 0 & 0 & 0 & 1 & -1 \\
0 & 0 & 0 & 0 & 0 & -1 & 1
\end{array}\right)
$$

Using the Algorithm DN in [34] (see Appendix B), it is easy to compute the order of a maximal-order positive-definite principle minor of matrix $\widetilde{L}_{1}$ in Theorem 2 is 5. As a result, the Algorithm DN will also indicates that one pair of nodes (such as nodes 5 and 6, or nodes 5 and 7, or nodes 1 and 7 and so on) need to be pinned to achieve the absolute stability of model (12). Given an initial vector value (here, the initial value of every node is chosen to be arbitrarily different) for the network (2), the corresponding state trajectories of system (2) are shown in Fig. 4. one can find the corresponding initial vector value of model (12) from that of system (2), and the state trajectories of system (12) without controller are shown in Fig. 5 .

If nodes 5 and 6 in model (12) are pinned, i.e., the diagonal matrix $\bar{D}=\operatorname{diag}(0,0,0,0,4,3,0)^{T}$, one can obtain the absolute stability of model (12). The corresponding state trajectories of system (12) are shown in Fig. 6. If the corresponding nodes in model (2) are pinned, one can obtain the absolute consensus of system (4). The state trajectories of system (4) are shown in Fig. 7. Consequently, the results in Theorem 2 are effective.

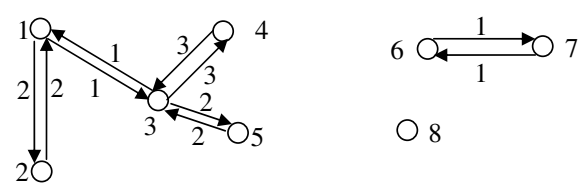

Figure 3: The aggregated graph of system (2) with 74 nodes.

\section{Conclusions}

In this paper, we have shed some lights on the pinning consensus of a MNDN. Based on the Lie algebra theory, a node-and-node linear pinning scheme has been introduced to achieve the absolute consensus of a MNDN. Based on some optimal algorithms, large-size networks have first been aggregated to small-size ones. Then, by using the principle minor theory to small-size networks effectively, another new pinning strategy has been proposed to achieve the absolute consensus of a MNDN. Finally, numerical examples with the computer simulations have been provided to illustrate the effectiveness of the obtained criteria. 


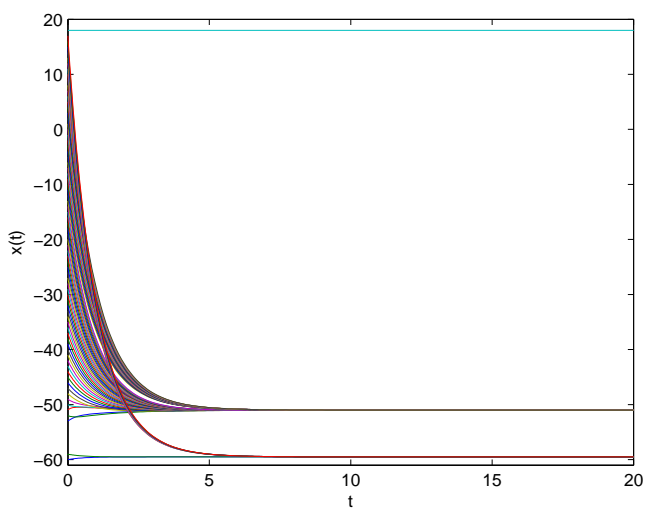

Figure 4: The numeric simulation of state variable $x(t)$ of system (2) in Example 2.

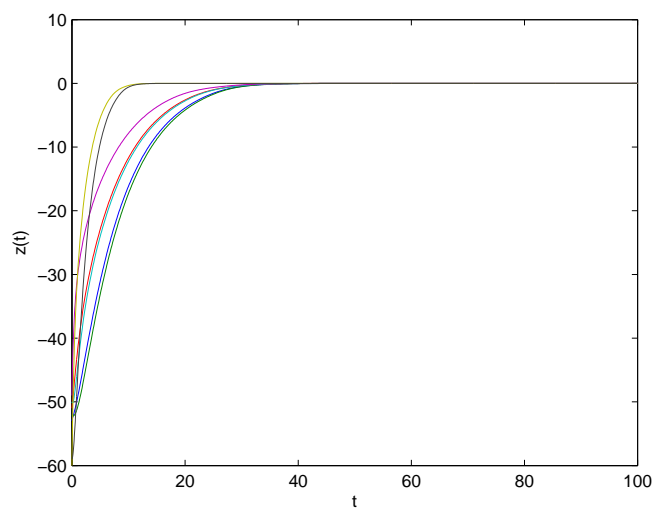

Figure 6: The numeric simulation of state variable $z(t)$ of system (12) in Example 2 with the proposed pinning scheme.

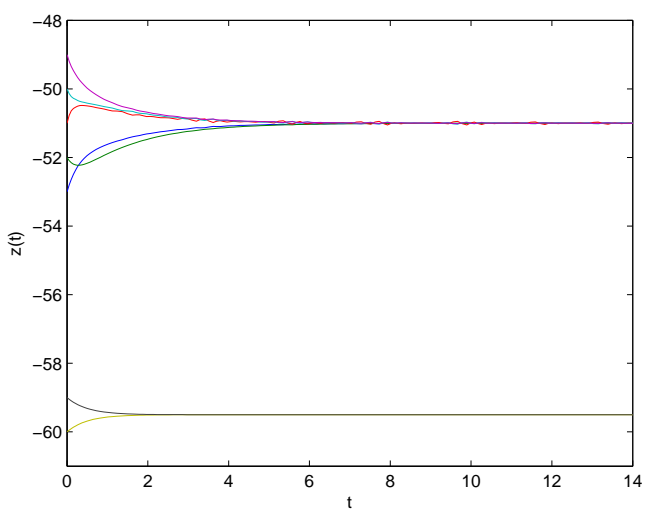

Figure 5: The numeric simulation of state variable $z(t)$ of system (12) without controllers.

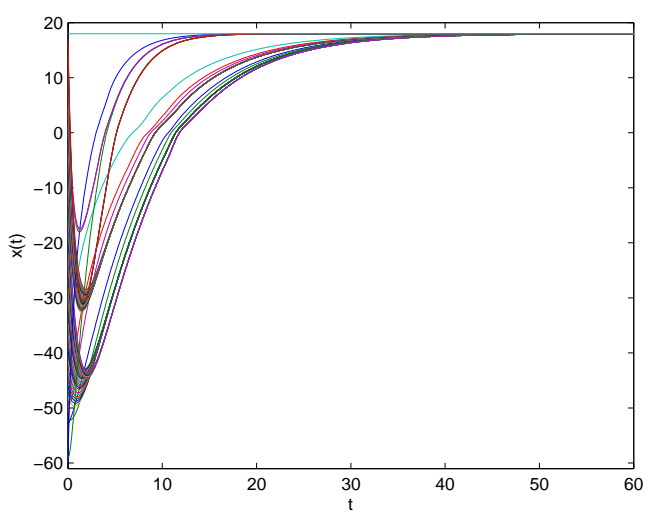

Figure 7: The numeric simulation of state variable $x(t)$ of system (4) in Example 2 with the proposed pinning scheme. 


\section{Appendix A}

Here, some details are shown to answer the question $Q_{1}$.

According to Lemma 3, without loss of generality, we suppose that $\mathfrak{L}^{(0)}$ only consists of upper triangular matrices. For all $A, B \in \mathfrak{L}^{(0)}$, both $A B$ and $B A$ are upper triangular and they have the same diagonal elements. Hence, the diagonal elements of the commutator product $[A, B]$ are zero and $\mathfrak{L}^{(1)}$ consists of upper triangular matrices with zero main diagonal, i.e.,

$$
\mathfrak{L}^{(1)}=\left\{A \mid A=\left(a_{i j}\right)_{n \times n}, \text { with } a_{i j}=0, \text { for } 1 \leq j \leq i \leq n\right\}
$$

Analogously,

$$
\mathfrak{L}^{(k)}=\left\{A \mid A=\left(a_{i j}\right)_{n \times n}, \text { with } a_{i j}=0, \text { for } 1 \leq j \leq i+k-1\right\}, \quad k=1,2,3, \cdots
$$

We shall apply the mathematical induction to prove (20). Suppose that (20) is true for all $k \leq N, N$ is a positive integer.

When $k=N+1, \forall A, B \in \mathfrak{L}^{(N)}$, letting $A B=M=\left(m_{i j}\right)_{n \times n}$, for $j \leq i+N$, we have

$$
\begin{aligned}
m_{i j} & =\sum_{s=1}^{n} a_{i s} B_{s j} \\
& =\sum_{s=1}^{i+N-1} a_{i s} B_{s j}+\sum_{s=i+N}^{n} a_{i s} B_{s j}=0+0=0 .
\end{aligned}
$$

Let $B A=\bar{M}=\left(\bar{m}_{i j}\right)_{n \times n}$. Similarly, it follows that

$$
\bar{m}_{i j}=0, \text { for } j \leq i+N
$$

namely,

$$
\mathfrak{L}^{(N+1)}=\left\{A \mid A=\left(a_{i j}\right)_{n \times n}, \text { with } a_{i j}=0, \text { for } 1 \leq j \leq i+N\right\}
$$

Hence, (20) is true. By induction, all matrices of $\mathfrak{L}^{(n)}$ are zero, i.e.,

$$
\mathfrak{L}^{(n)}=\{0\} .
$$

\section{Appendix B}

\section{Algorithm DN (see [34])}

1) For matrix $G$, calculate the eigenvalues of matrices $G$, and find the number of non-positive eigenvalues $k$.

2) Set $j:=k$. (Here, $j$ is the number of nodes to be pinned. Clearly, $j \geq k$. That is, the initial choice of $j$ is the number (i.e., $k$ ) of non-positive eigenvalues of $G$.) 
3) If $G$ contains a $(n-j)$-order positive definite principle minor, then the number of pinned nodes will be $j$.

- The $j$ nodes which are not included in the $(n-j)$-order positive definite principle minor should be pinned.

- Note that the $(n-j)$-order positive definite principle minor may not be unique. Therefore, there

may be more than one set of $j$ nodes which can be pinned. One may only choose one of those sets to be the pinned nodes and stop the process.

- Stop the whole process when $j=n$. Here, $n$ nodes should be all pinned.

4) If $G$ does not contain a $(n-j)$-order positive definite principle minor, then set $j:=k+1$ (i.e., increase the number of nodes to be pinned). Go to step 3 .

\section{REFERENCES}

[1] D. Lee and M. W. Spong, "Stable flocking of multiple inertial agents on balanced graphs," IEEE Transactions on Automatic Control, vol. 52, no. 8, pp. 1469-1475, 2007.

[2] T. Vicsek, "A question of scale," Nature, vol. 411, no. 6836, p. 421, 2001.

[3] D. Low, "Following the crowd," Nature, vol. 407, no. 1, pp. 465-466, 2000.

[4] M. DeGroot, "Reaching a consensus," Journal of the American Statistical Association, pp. 118-121, 1974.

[5] A. Jadbabaie, J. Lin, and A. Morse, "Coordination of groups of mobile autonomous agents using nearest neighbor rules," IEEE Transactions on Automatic Control, vol. 48, no. 6, pp. 988-1001, 2003.

[6] L. Moreau, "Stability of multiagent systems with time-dependent communication links," IEEE Transactions on Automatic Control, vol. 50, no. 2, pp. 169-182, 2005.

[7] R. Olfati-Saber and R. Murray, "Consensus problems in networks of agents with switching topology and time-delays," IEEE Transactions on Automatic Control, vol. 49, no. 9, pp. 1520-1533, 2004.

[8] W. Yang, L. Cao, X. Wang, and X. Li, "Consensus in a heterogeneous influence network," Physical Review E, vol. 74, no. 3, p. 037101, 2006.

[9] Y. Liu, Z. Wang, J. Liang, and X. Liu, "Synchronization and state estimation for discrete-time complex networks with distributed delays," IEEE Transactions on Systems, Man, and Cybernetics-B, vol. 38, no. 5, pp. 1314-1325, 2008.

[10] W. Wu and T. Chen, "Global synchronization criteria of linearly coupled neural network systems with time-varying coupling," IEEE Transactions on Neural Networks, vol. 19, no. 2, pp. 319-332, 2008.

[11] W. Yu, J. Cao, and J. Lü, "Global synchronization of linearly hybrid coupled networks with timevarying delay," SIAM Journal on Applied Dynamical Systems, vol. 7, pp. 108-133, 2008. 
[12] J. Liang, Z. Wang, Y. Liu, and X. Liu, "Robust synchronization of an array of coupled stochastic discrete-time delayed neural networks," IEEE Transactions on Neural Networks, vol. 19, no. 11, pp. 1910-1921, 2008.

[13] W. Ren and R. Beard, "Consensus seeking in multiagent systems under dynamically changing interaction topologies," IEEE Transactions on Automatic Control, vol. 50, no. 5, pp. 655-661, 2005.

[14] S. Bohte, H. La Poutre, and J. Kok, "Unsupervised clustering with spiking neurons by sparse temporal coding and multilayer RBF networks," IEEE Transactions on Neural Networks, vol. 13, no. 2, pp. 426$435,2002$.

[15] W. Yu, G. Chen, Z. Wang, and W. Yang, "Distributed consensus filtering in sensor networks," IEEE Transactions on Systems, Man, and Cybernetics-B, vol. 39, no. 6, pp. 1568-1577, 2009.

[16] P. Lin, Y. Jia, and L. Li, "Distributed robust $H_{\infty}$ consensus control in directed networks of agents with time-delay," Systems \& Control Letters, vol. 57, no. 8, pp. 643-653, 2008.

[17] J. Cao, G. Chen, and P. Li, "Global synchronization in an array of delayed neural networks with hybrid coupling," IEEE Transactions on Systems Man and Cybernetics-B, vol. 38, no. 2, pp. 488-498, 2008.

[18] J. Cao and L. Li, "Cluster synchronization in an array of hybrid coupled neural networks with delay," Neural Networks, vol. 22, no. 4, pp. 335-342, 2009.

[19] C. Wu, "Synchronization in networks of nonlinear dynamical systems coupled via a directed graph," Nonlinearity, vol. 18, no. 3, pp. 1057-1064, 2005.

[20] X. Liu, T. Chen, and W. Lu, "Consensus problem in directed networks of multi-agents via nonlinear protocols," Physics Letters A, vol. 373, no. 35, pp. 3122-3127, 2009.

[21] R. O. Grigoriev, M. C. Cross, and H. G. Schuster, "Pinning control of spatiotemporal chaos," Physical Review Letters, vol. 79, no. 15, pp. 2795-2798, 1997.

[22] N. Parekh, S. Parthasarathy, and S. Sinha, "Global and local control of spatiotemporal chaos in coupled map lattices," Physical Review Letters, vol. 81, no. 7, pp. 1401-1404, 1998.

[23] X. F. Wang and G. Chen, "Pinning control of scale-free dynamical networks," Physica A, vol. 310, no. 3-4, pp. 521-531, 2002.

[24] T. Chen, X. Liu, and W. Lu, "Pinning complex networks by a single controller," IEEE Transactions on Circuits and Systems-I, vol. 54, no. 6, pp. 1317-1326, 2007.

[25] W. Yu, G. Chen, and J. Lü, "On pinning synchronization of complex dynamical networks," Automatica, vol. 45, no. 2, pp. 429-435, 2009.

[26] J. Xiang and G. Chen, "On the V-stability of complex dynamical networks," Automatica, vol. 43, no. 6, pp. 1049-1057, 2007. 
[27] W. Lu, X. Li, and Z. Rong, "Global stabilization of complex networks with digraph topologies via a local pinning algorithm," Automatica, vol. 46, no. 1, pp. 116-121, 2010.

[28] M. Porfiri and F. Fiorilli, "Node-to-node pinning control of complex networks," Chaos, vol. 19, p. 013122, 2009.

[29] X. Li, X. Wang, and G. Chen, "Pinning a complex dynamical network to its equilibrium," IEEE Transactions on Circuits and Systems-I, vol. 51, no. 10, pp. 2074-2087, 2004.

[30] J. Lu, D. Ho, and Z. Wang, "Pinning stabilization of linearly coupled stochastic neural networks via minimum number of controllers," IEEE Transaction Neural Networks, vol. 20, no. 10, pp. 1617-1629, 2009.

[31] W. Xia and J. Cao, "Pinning synchronization of delayed dynamical networks via periodically intermittent control," Chaos, vol. 19, p. 013120, 2009.

[32] Y. Tang, Z. Wang, and J. Fang, "Pinning control of fractional-order weighted complex networks," Chaos, vol. 19, no. 1, p. 013112, 2009.

[33] F. Chen, Z. Chen, L. Xiang, Z. Liu, and Z. Yuan, "Reaching a consensus via pinning control," Automatica, vol. 45, no. 5, pp. 1215-1220, 2009.

[34] W. Xiong, D. W. C. Ho, and C. Huang, "Pinning synchronization of time-varying polytopic directed stochastic networks," Physics Letters A, vol. 374, no. 3, pp. 439-447, 2010.

[35] A. A. Sagle and R. E. Walde, Introduction to Lie groups and Lie algebras. New York: Academic Press, 1973.

[36] T. Chu and C. Zhang, "New necessary and sufficient conditions for absolute stability of neural networks," Neural Networks, vol. 20, no. 1, pp. 94-101, 2007.

[37] C. Wu, I. Center, and N. Yorktown Heights, "Agreement and consensus problems in groups of autonomous agents with linear dynamics," in IEEE International Symposium on Circuits and Systems, pp. 292-295, 2005.

[38] H. Ishii, R. Tempo, E.-W. Bai, and F. Dabbene, "Distributed Randomized PageRank Computation Based on Web Aggregation," in Joint 48th IEEE Conference on Decision and Control and 28th Chinese Control Conference, pp. 3026-3031, 2009. 